\title{
Dirac and Klein-Gordon Equations: Convergence of Solutions in the Nonrelativistic Limit
}

\author{
Ross J. Cirincione and Paul R. Chernoff * \\ Department of Mathematics, University of California, Berkeley, CA 94720, USA
}

\begin{abstract}
The convergence of solutions of the Dirac and Klein-Gordon equations to solutions of the Pauli and Schrödinger equations in the nonrelativistic limit is discussed. An abstract theory of these equations is developed which is general enough to allow physical space to be an arbitrary complete Riemannian manifold.
\end{abstract}

\section{Introduction}

Our object is to discuss the sense in which solutions of relativistic wave equations approximate solutions of the corresponding non-relativistic Schrödinger equations when the speed of light tends to infinity. We are concerned specifically with the Dirac and Klein-Gordon equations for a particle in an external electromagnetic field. The problem, at least on a formal level, has a long history, but rigorous results have a rather recent provenance. Veselic $[11,12]$ dealt with spectral convergence and analyticity of eigenfunctions in $1 / c$. His results for the Dirac operator were improved and extended by Hunziker [6]. Veselic also treated the Klein-Gordon equation, but only under extremely restrictive conditions on the electric potential, with vanishing magnetic potential. These authors were concerned with resolvent convergence, and they showed that in some sense the resolvent of the relativistic Hamiltonian converges to that of the non-relativistic Hamiltonian as $c$ tends to $\infty$.

Once one has resolvent convergence, the Trotter-Kato theorem may be used to deduce convergence of the corresponding one-parameter groups, and hence convergence of solutions of the equations. We follow a variant of this approach, using a generalization of the Trotter-Kato theorem formulated by Davies [4].

In Sect. 2 we discuss an abstract version of the Dirac equation with relatively bounded potentials, simplifying and also generalizing Hunziker's calculations. In Sect. 3 the abstract theory is applied to some concrete cases, including Dirac operators over curved spaces. In Sect. 4 we outline a parallel discussion of the

* Research partially supported by National Science Foundation grant MCS-77-13070 
Klein-Gordon equation. Many of the results of this paper are contained, in somewhat different form, in [3].

\section{An Abstract Dirac Equation}

We shall consider the nonrelativistic limit of an abstract Dirac equation which is general enough to encompass the case of curved space as well as the usual Dirac equation over $\mathbb{R}^{3}$ or $\mathbb{R}^{n}$. To this end, we will work in a setting which is somewhat more general than that considered by Hunziker [6]. In Sect. 3 we will indicate how the concrete special cases fit into this abstract approach.

Let $\mathbb{H}$ be a Hilbert space and let $A$ and $\beta$ be two self-adjoint operators on $\mathbb{H}$ with the following commutation properties:

$$
\begin{gathered}
\beta^{2}=I \\
A \beta+\beta A=0 \text { or } A=-\beta A \beta .
\end{gathered}
$$

These formulas are what remain of Dirac's anticommutation relations in our setting. Note that (2.1) implies that $\beta$ is bounded (and also unitary as well as self-adjoint). The operator $A$ will be unbounded in all interesting cases.

The operators $A, \beta$ can be represented more concretely by two-by-two operator matrices. Indeed, since $\beta^{2}=I$, the operator $\beta$ has two spectral projections $P_{+}, P_{-}$ corresponding to the eigenvalues $+1,-1$. Note the formula $P_{ \pm}=(1 \pm \beta) / 2$. $\mathbb{H}$ is the orthogonal direct sum $\mathbb{H}_{+} \oplus \mathbb{H}_{-}$, where $\mathbb{H}_{ \pm}$is the range of $P_{ \pm}$. With respect to this decomposition we can represent $A$ and $\beta$ by operator matrices. We have

$$
\beta=\left[\begin{array}{rr}
I & 0 \\
0 & -I
\end{array}\right], \quad A=\left[\begin{array}{ll}
A_{11} & A_{12} \\
A_{21} & A_{22}
\end{array}\right]
$$

where $A_{11}$ and $A_{22}$ are self-adjoint while $A_{12}, A_{21}$ are closed and densely defined with $A_{21}=A_{12}^{*}$. The condition $A \beta=-\beta A$ means that $A_{11}=A_{22}=0$, so that $A$ is of the form

$$
A=\left[\begin{array}{cc}
0 & A_{0} \\
A_{0}^{*} & 0
\end{array}\right]
$$

where $A_{0}: \mathbb{H}_{-} \rightarrow \mathbb{H}_{+}$is a closed, density defined operator with adjoint $A_{0}^{*}: \mathbb{H}_{+} \rightarrow \mathbb{H}_{-}$. If, for example, $\mathbb{H}_{+}=\mathbb{H}_{-}=\mathbb{H}_{0}$, a given Hilbert space, so that $\mathbb{H} \cong \mathbb{C}^{2} \otimes \mathbb{H}_{0}$, and if $A_{0}=A_{0}^{*}$, then we have $A=\alpha \otimes A_{0}$, where $\alpha$ is the two-by-two matrix $\left[\begin{array}{ll}0 & 1 \\ 1 & 0\end{array}\right]$. This is the case which Hunziker discusses. However, as we will see in Sect. 3, the matrix representation is not always very natural, and the more abstract approach does in fact have some practical value.

Our abstract "free Dirac operator" is defined by

$$
H_{0}(c)=c A+m c^{2} \beta \text {. }
$$

Here $m$ and $c$ are positive constants which we call the "rest mass" and the "velocity 
of light." Because of the commutation relations (2.1) and (2.2), we have the "Einstein relation"

$$
H_{0}(c)^{2}=c^{2} A^{2}+m^{2} c^{4}
$$

so that the spectrum of $H_{0}(c)$ has a gap from $-m c^{2}$ to $+m c^{2}$. Let $P_{+}(c)$ and $P_{-}(c)$ be the spectral projections corresponding to the positive and negative pieces of the spectrum of $H_{0}(c)$. Then, because of the formulas

$$
\begin{aligned}
P_{+}(c)+P_{-}(c) & =I \\
P_{+}(c)-P_{-}(c) & =\left[H_{0}(c)^{2}\right]^{-1 / 2} H_{0}(c) \\
& =\left(c^{2} A^{2}+m^{2} c^{4}\right)^{-1 / 2}\left(A+m c^{2} \beta\right),
\end{aligned}
$$

it is straightforward to show that when $c \rightarrow \infty P_{ \pm}(c)$ converges strongly to $P_{ \pm}(\infty)=$ $P_{ \pm}=(1 \pm \beta) / 2$.

Using the spectral theorem, it is not difficult to show that

$$
e^{ \pm i t m c^{2}} e^{i t H_{0}(c)} P_{ \pm}(c) \rightarrow e^{ \pm i t\left(A^{2}\right)_{ \pm} / 2 m} P_{ \pm}(\infty)
$$

in the strong operator topology, uniformly on bounded $t$-intervals, as the speed of light $c$ tends to $\infty$. Here $\left(A^{2}\right)_{+}=A_{0} A_{0}^{*}$ and $\left(A^{2}\right)_{-}=A_{0}^{*} A_{0}$ are the restrictions of $A^{2}$ to the invariant subspaces $\mathbb{H}_{ \pm}, \mathbb{H}_{-}$. In this sense solutions of the free Dirac equation tend to solutions of the free Schrödinger equation. It is important to note the correction factors $e^{ \pm i t m c^{2}}$ and the splitting of the positive and negative energy solutions in (2.6).

Next we introduce an operator $V$, representing a potential, and define

$$
H(c)=H_{0}(c)+V=c A+m c^{2} \beta+V .
$$

We require that $V$ be self-adjoint, bounded relative to $A$, and $V \beta=\beta V$. The latter condition means that $V$ is of the form $\left[\begin{array}{cc}V_{+} & 0 \\ 0 & V_{-}\end{array}\right]$where $V_{+}, V_{-}$are selfadjoint operators on $\mathbb{H}_{+}, \mathbb{H}_{-}$. (Usually $\mathbb{H}_{+}=\mathbb{H}_{-}$and $V_{+}=V_{-}$.) Since $V$ is bounded relative to $A$, if $c$ is sufficiently large $V$ will be bounded relative to $H_{0}(c)$ with relative bound less than 1 . Then $H(c)=H_{0}(c)+V$ will be self-adjoint by the Kato-Rellich theorem. Henceforth we will assume that $c$ is large enough so that this is the case. Note also that $A^{2} / 2 m+V$ is a self-adjoint operator. For our hypotheses on $V$ guarantee that $V$ is an infinitesimal Kato perturbation of $A^{2}$. Hence the operators

$$
H^{ \pm}(\infty)=\left(A^{2}\right)_{ \pm} / 2 m+V_{ \pm}
$$

are self-adjoint on the spaces $\mathbb{H}_{ \pm}, H_{-}$.

Lemma 2.1. Given any vector $f$ in the domain of $H^{+}(\infty)$, there exist vectors $\left[\begin{array}{l}f(c) \\ g(c)\end{array}\right]$ in the domain of $H(c)$ such that, as $c \rightarrow \infty$,

$$
\left[\begin{array}{l}
f(c) \\
g(c)
\end{array}\right] \rightarrow\left[\begin{array}{l}
f \\
0
\end{array}\right]
$$


and

$$
\left(H(c)-m c^{2}\right)\left[\begin{array}{l}
f(c) \\
g(c)
\end{array}\right] \rightarrow\left[\begin{array}{c}
H^{+}(\infty) f \\
0
\end{array}\right]
$$

Proof. Note that $D\left(H^{+}(\infty)\right)=D\left(A_{0} A_{0}^{*}\right)$. For $f$ in this domain we set

$$
f(c)=f, \quad g(c)=A_{0}^{*} f / 2 m c .
$$

Then (2.7a) is automatic. As for (2.7b), we use the matrix representation

$$
H(c)-m c^{2}=\left[\begin{array}{cc}
V_{+} & c A_{0} \\
c A_{0}^{*} & V_{-}-2 m c^{2}
\end{array}\right] \text {. }
$$

Applied to the vector $[f(c), g(c)]$ this yields $\left[V_{+} f+\frac{1}{2 m} A_{0} A_{0}^{*} f, \frac{1}{2 m c} V_{-} A_{0}^{*} f\right]$, so that $(2.7 b)$ is evident.

With this in hand, we may apply Davies' generalization of the Trotter-Kato semigroup convergence theorem [4, Theorem 1.1] to deduce that

$$
e^{-i t m c^{2}} e^{i t H(c)} P^{+}(\infty) \rightarrow e^{i t H^{+}(\infty)} P^{+}(\infty)
$$

strongly, uniformly on bounded $t$-intervals, as $c \rightarrow \infty$. (We also get an analogous result with the + signs replaced by - signs.)

Next, we examine the behavior of the spectral projections associated with $H(c)$. We need the following simple estimate.

Lemma 2.2. For $\psi$ in the domain of $A$,

$$
\|V \psi\|^{2} \leqq a(c)\left\|H_{0}(c) \psi\right\|^{2}+b(c)\|\psi\|^{2}
$$

where the constants $a(c), b(c)$ tend to 0 as $c \rightarrow \infty$.

Proof. Since $V$ is bounded relative to $A$, there are constants $a_{0}, b_{0}$ such that

$$
\|V \psi\|^{2} \leqq a_{0}\|A \psi\|^{2}+b_{0}\|\psi\|^{2}
$$

for $\psi$ in $\mathscr{D}(A)$. Now we expand:

$$
\begin{aligned}
\left\|H_{0}(c) \psi\right\|^{2} & =\left(\left(c A+m c^{2} \beta\right) \psi,\left(c A+m c^{2} \beta\right) \psi\right) \\
& =c^{2}\left\|A^{2} \psi\right\|+m^{2} c^{4}\|\psi\|^{2}+m c^{3}[(A \psi, \beta \psi)+(\beta \psi, A \psi)] \\
& =c^{2}\left\|A^{2} \psi\right\|+m^{2} c^{4}\|\psi\|^{2}
\end{aligned}
$$

where we have used the commutation relation (2.2). Accordingly the assertion of the lemma follows, with $a(c)$ proportional to $1 / c^{2}$ and $b(c)$ identically 0 .

The estimate (2.9) of Lemma 2.2 allows us to apply a theorem of Heinz (see Kato [7, Theorem VI.5.12]) concerning the spectrum of $H(c)=H_{0}(c)+V$. Namely, if $c$ is sufficiently large, then the spectrum of $H(c)$ has a gap containing 0 . Moreover, if $Q^{+}(c), Q^{-}(c)$ are the spectral projections corresponding to the positive and negative halves of the spectrum of $H(c)$, then $\left\|Q^{+}(c)-P^{+}(c)\right\|$ and $\left\|Q^{-}(c)-P^{-}(c)\right\|$ both tend to 0 as $c \rightarrow \infty$. Here $P^{+}(c), P^{-}(c)$ are the corresponding projections associated with $H_{0}(c)$. (Note: The cited theorem in Kato's book refers to pertur- 
bations of a fixed operator, but it is easy to see that the estimates given there are uniform in $c$.)

Now, from (2.5), we know that the projections $P^{ \pm}(c)$ converge strongly to $P_{ \pm}(\infty)$ as $c \rightarrow \infty$. Conclusions:

$$
Q^{ \pm}(c) \rightarrow P^{ \pm}(\infty)
$$

strongly as $c \rightarrow \infty$.

To summarize:

Theorem 2.3. Let $H(c)=c A+m c^{2} \beta+V$ where $V$ commutes with $\beta$ and is bounded relative to $A$ and $A, \beta$ satisfy (2.1), (2.2). Then, for $c$ sufficiently large, $H(c)$ is selfadjoint and has a gap in its spectrum containing 0 . The corresponding spectral projections $Q^{ \pm}(c)$ converge strongly to $P^{ \pm}(\infty)=(1 \pm \beta) / 2$ as $c \rightarrow \infty$. Moreover, we have

$$
e^{\mp i t m c^{2}} e^{i t H(c)} Q^{ \pm}(c) \rightarrow e^{ \pm i t H^{ \pm}(\infty)} P^{ \pm}(\infty)
$$

strongly, uniformly on bounded t-intervals, as $c \rightarrow \infty$. Here

$$
H^{ \pm}(\infty)=\left(A^{2}\right)_{ \pm} / 2 m+V_{ \pm}
$$

where the suffixes,+- denote restrictions to $\mathbb{H}_{+}, \mathbb{H}_{-}$, the ranges of $P^{+}(\infty)$ and $P^{-}(\infty)$.

Remark. It is important to note that Theorem 2.3 enables us to deal with "magnetic fields" as well as "electrostatic fields" $V$ with no additional effort. Indeed, as we shall see in detail in Sect. 3, a magnetic potential is simply a perturbation $B$ which anticommutes with $\beta$, so we may regard $B$ as a perturbation of $A$. To be precise, assume that $B$ is a self-adjoint operator on $\mathbb{H}$ which anticommutes with $\beta$ and which is bounded relative to $A$ with relative bound less than 1 . Then $V$ is bounded relative to the self-adjoint operator $A+B$, so that

$$
H(c)=c(A+B)+m c^{2} \beta+V
$$

is self adjoint for sufficiently large $c$, and the preceding theory applies verbatim with $A+B$ replacing $A$. The Schrödinger Hamiltonian is replaced by the "Pauli" Hamiltonian

$$
H(\infty)=(A+B)^{2} / 2 m+V,
$$

and all our results about resolvent and unitary group convergence apply to this case. (This observation enables many of the complications in [6] to be dispensed with.)

\section{Applications: Concrete Dirac Operators}

The abstract theory developed in the preceding section may be applied to a number of concrete special cases. Among these are the usual Dirac operator over flat space as well as more general operators of Dirac type over Riemannian manifolds. 


\section{A. The Standard Dirac Operator}

This is the case discussed by Hunziker [6]. The Hilbert space $\mathbb{H}$ is $L^{2}\left(\mathbb{R}^{3} ; \mathbb{C}^{4}\right)$, which we may write as $\mathbb{C}^{2} \otimes L^{2}\left(\mathbb{R}^{3} ; \mathbb{C}^{2}\right)$. The Dirac Hamiltonian is given by

$$
H(c)=c \alpha \otimes\left(\boldsymbol{\sigma} \cdot(i \nabla-\mathbf{A}(x))+m c^{2} \beta+V(x)\right.
$$

where $\nabla$ is the gradient operator, $\sigma_{1}, \sigma_{2}, \sigma_{3}$ are the Pauli spin matrices, and $\alpha, \beta$ are the two-by-two matrices discussed in Sect. 3. $\mathbf{A}(x)$ is the magnetic vector potential and $V(x)$ is the electric potential. (Our units are chosen so that $\hbar=e=1$ where $e$ is the electric charge.) The theory of Sect. 3 will be applicable provided that the multiplication operator $\boldsymbol{\sigma} \cdot \mathbf{A}(x)$ is bounded relative to $\boldsymbol{\sigma} \cdot \boldsymbol{\nabla}$ with relative bound less than one, and also the operator $V(x)$ is bounded relative to $\sigma \cdot \nabla$. It is well known that this will be so provided $\mathbf{A} \in L^{p}+L^{\infty}$ for some $p>3$ (cf. Prosser [9]), while $V$ may be of the form $V_{1}+V_{2}+V_{3}$ where $V_{1} \in L^{p}, p>3, V_{2} \in L^{\infty}$, and $V_{3}$ is a sum of Coulomb terms (cf. Kato [7, V.5.10]). Then the non-relativistic Hamiltonian is the Pauli Hamiltonian

$$
H(\infty)=\left(\boldsymbol{\sigma} \cdot(i \nabla-\mathbf{A}(x))^{2}+V(x)\right.
$$

acting on $L^{2}\left(\mathbb{R}^{2}, \mathbb{C}^{3}\right)$. (In this case $H_{+}(\infty)$ and $H_{-}(\infty)$ are the same.)

\section{B. Operators over Riemannian Manifolds}

Let $M$ be a finite dimensional $C^{\infty}$ Riemannian manifold, and let $\xi$ be a Hermitian vector bundle over $M-$ a complex vector bundle equipped with a smoothly varying inner product on each fiber $\xi_{x}$. We will work with operators on the Hilbert space $\mathbb{H}=L^{2}(\xi)$ of square-integrable sections of $\xi$ with the inner product

$$
(\phi, \psi)=\int_{M}\langle\phi(x), \psi(x)\rangle d V
$$

where $d V$ is the Riemannian volume element.

A first-order differential operator $L$ can be expressed in local coordinates by

$$
L \phi=\sum_{i=1}^{n} A_{i}(x) \frac{\partial \phi}{\partial x_{i}}+B(x) \phi
$$

where the coefficients are matrix functions of $x$.

We recall that the symbol $\sigma$ of $L$ is defined as follows (cf. Palais [8]): for each point $x \in M$ and cotangent vector $v \in T_{x}^{*} M$ we have a linear map $\sigma(v, x)$ on the fiber $\xi_{x}$ given by the formula

$$
\sigma(v, x) e=L(g \phi)(x)-g(x)(L \phi)(x)
$$

where $\phi \in C^{\infty}(\xi)$ is any smooth section of $\xi$ such that $\phi(x)=a$, and $g \in C^{\infty}(M)$ is any smooth function with differential $d g(x)=v$. In local coordinates we have

$$
\sigma(v, x)=\sum_{i=1}^{n} v^{i} A_{i}(x)
$$

For example, if $L$ is the usual Dirac operator $\mathbb{R}^{3}$, we have the formula 


$$
\sigma(v, x)=\sigma(v)=v^{1} \alpha_{1}+v^{2} \alpha_{2}+v^{3} \alpha_{3}
$$

where the $\alpha_{i}$ are the four-by-four Dirac matrices: $\alpha_{i}=\alpha \otimes \sigma_{i}$.

Definition. By a Dirac-type pair of operators we mean a first-order linear differential operator $D$ on $L^{2}(\xi)$ together with a zeroth order operator $\beta$ such that the following conditions are satisfied:

(i) $D$ is formally skew-symmetric; that is, $(D \phi, \psi)=-(\phi, D \psi)$ for any sections $\phi, \psi$ which are $C^{\infty}$ with compact support. Note that as a consequence the symbol $\sigma(v, x)$ is a symmetric operator on $\xi_{x}$.

(ii) The symbol $\sigma$ of $D$ obeys the Clifford algebra anticommutation relations:

$$
\sigma(v, x) \sigma(w, x)+\sigma(w, x) \sigma(v, x)=g_{x}(v, w)=\sum_{i j} g_{i j}(x) v^{i} w^{j}
$$

where $g$ is the Riemannian metric of $M$.

(iii) The parity operator $\beta$ is defined by $(\beta \phi)(x)=\beta_{x} \phi(x)$, where $\beta_{x}$ is a symmetric linear operator on $\xi_{x}$, varying smoothly with $x$, such that $\beta_{x}^{2}=I$. We require that $\beta D+D \beta=0$. In particular it follows that $\beta_{x}$ anticommutes with $\sigma(v, x)$ for every $v$ in $T_{x}^{*} M$.

Condition (iii) says that the vector bundle $\xi$ has an orthogonal direct sum decomposition $\xi=\xi^{+} \oplus \xi^{-}$such that $D$ maps sections of $\xi^{+}$into sections of $\xi^{-}$ and vice-versa. The operator $\beta$ is +1 on $\xi^{+},-1$ on $\xi^{-}$. D thus splits into two operators $D^{+}, D^{-}$where $D^{+}$maps $\xi^{+}$to $\xi^{-}$and $D^{-}$maps $\xi^{-}$to $\xi^{+}$.

A special case of $[1$, Theorem 2.2] is that if $M$ is a complete Riemannian manifold and $D$ is a Dirac-type operator over $M$, then the operator $i D$, with domain $C_{0}^{\infty}(\xi)$, is essentially self-adjoint on the Hilbert space $L^{2}(\xi)$. We can then define a free Dirac Hamiltonian of the sort considered in Sect. 2 by

$$
H_{0}(c)=c i D+m c^{2} \beta .
$$

We introduce lower-order perturbations corresponding to scalar and vector electromagnetic potentials as follows.

The scalar potential $V$ is given by multiplication by a function $V(x)$ defined on $M$. (More generally, $V(x)$ might be a symmetric linear map on $\xi_{x}$ which commutes with $\beta_{x}$.) For $V$ to be bounded relative to $i D$ it suffices that $V$ be locally in $L_{p}(M)$ for $p>n$ (the dimension of $M$ ) and that $V$ be bounded at infinity. The proof is essentially the same as that for $\mathbb{R}^{3}$ (cf. [9]).

Now suppose that $\mathbf{a}(x)$ is a vector field on $M$. Using the Riemannian metric $g$ we can regard a as a field of cotangent vectors (or a 1-form) on $M$. Then, for each $x$ in $M$, define

$$
\mathbf{A}(x)=\sigma(\mathbf{a}(x), x)
$$

where $\sigma$ is the symbol of $D$. Then, for each $x$ in $M, \mathbf{A}(x)$ is a symmetric operator on $\xi_{x}$ and $\mathbf{A}(x)$ anticommutes with $\beta_{x}$. We define $\mathbf{A}$ to be multiplication by $\mathbf{A}(x)$ on $\xi_{x}$. A is the operator corresponding to the vector potential a on $M$. Thus our Dirac Hamiltonian is

$$
H(c)=c \cdot(i D-A)+m c^{2} \beta+V .
$$

(The general question of self-adjointness for such operators is discussed in [2].) 


\section{The De Rham Operator}

This is perhaps the simplest example of a Dirac-type operator over a general Riemannian manifold $M$. Let $\xi$ be the complexified exterior bundle of the cotangent bundle $T^{*} M$; thus sections of $\xi$ are complex-valued differential forms. The Riemannian metric of $M$ induces a Hermitian structure on $\xi$. Let $d$ be exterior differentiation and let $\delta$ be its formal adjoint. Then $D=d-\delta$ is a formally skewsymmetric first order differential operator on $\xi$. The symbol $\sigma$ of $D$ is given by

$$
\sigma(v) e=v \wedge e+i_{v} e
$$

where $\wedge$ is the exterior product and $i$ is interior multiplication (cf. [18]). It is easy to verify the anticommutation relations

$$
\sigma(v) \sigma(w)+\sigma(w) \sigma(v)=v \cdot w .
$$

Note that $D^{2}=\Delta$, the Laplace-Beltrami operator for $M$. The operator $\beta$ is induced by space inversion on each cotangent space $T_{x}^{*} M$. Thus $\beta \psi=(-1)^{p} \psi$ if $\psi$ is a $p$-form. It follows immediately that $\beta$ anti-commutes with $d$ and $\delta$ separately, hence with $D$. The bundle $\xi$ splits into $\xi^{+} \oplus \xi^{-}$where $\xi^{+}=$forms of even degree, $\xi^{-}=$forms of odd degree. Accordingly, $(D, \beta)$ is a Dirac-type pair in the sense of our definition. The corresponding "Dirac equation" is really a generalization of the Maxwell equations; the case of 3-dimensional space illustrates this most clearly.

\section{The Dirac Operator for a Spin Manifold}

Suppose that $M$ is an $n$-dimensional oriented Riemannian manifold with a spin structure - a reduction of the structural group of the tangent bundle $T(M)$ from $\mathrm{SO}(n)$ to its double covering group $\operatorname{Spin}(n)$ (cf. [8, Sect. 10]). The group $\operatorname{Spin}(n)$ has a unitary representation $S_{n}$ of dimension $2^{m}$, where $n=2 m$ or $2 m+1$, gotten by considering the action of $\operatorname{Spin}(n)$ on the Clifford algebra of $\mathbb{R}^{n}$. This representation is called the spin representation. If $n=2 m+1$ is odd, then $S_{n}$ is irreducible. On the other hand, if $n=2 m$ is even, then $S_{n}$ decomposes into a sum $S_{n}^{+} \oplus S_{n}$ of irreducible subrepresentations of dimension $2^{m-1}$ called the half-spin representations of Spin $(2 m)$. (For details see [14, Sect. 1].) Suppose now that $n$ is even, and let $\mathscr{S}$ be the bundle associated with the spin representation $S_{n}$, so that $\mathscr{S}=$ $\mathscr{S}^{+} \oplus \mathscr{S}^{-}$where $\mathscr{S}^{+}, \mathscr{S}^{-}$are associated with the two half-spin representations. The Dirac operator $D$ maps $\mathscr{S}^{+}$to $\mathscr{S}^{-}$and $\mathscr{S}^{-}$to $\mathscr{S}^{+}$; it is a first order skewsymmetric differential operator on $\mathscr{S}$ whose symbol is $\sigma(v)=$ Clifford multiplication by $v$; in local coordinates,

$$
D=\sum_{i=1}^{n} e_{i} \cdot \nabla_{e_{i}}
$$

where $\left\{e_{i}\right\}$ is a local orthonormal frame, $\nabla$ is covariant differentiation associated with the Riemannian metric, and the dot denotes Clifford multiplication. The operator $\beta$ is +1 on $\mathscr{S}^{+},-1$ on $\mathscr{S}^{-}$.

Odd-dimensional manifolds are less interesting. We can still define a Dirac 
operator $D_{0}$ on the spin bundle $\mathscr{S}$, but we then have to take $\xi=\mathscr{S} \oplus \mathscr{S}$ with $D=\left[\begin{array}{cc}0 & D_{0} \\ D_{0} & 0\end{array}\right]$ and $\beta=\left[\begin{array}{rr}1 & 0 \\ 0 & -1\end{array}\right]$. (If $M=\mathbb{R}^{3}, D_{0}$ is the Pauli operator $\sum_{1}^{3} \sigma_{i} \frac{\partial}{\partial x_{i}}$ as discussed in $(A)$.)

\section{The Klein - Gordon Equation}

The Klein-Gordon equation for a relativistic particle of spin zero and mass $m$ in an external electromagnetic field $(V, \mathbf{A})$ is

$$
\left(i \frac{\partial}{\partial t}+V(x)\right)^{2} \psi=\sum_{k=1}^{3} c^{2}\left(\frac{1}{i} \frac{\partial}{\partial x_{k}}-A_{k}(x)\right)^{2} \psi+m^{2} c^{4} \psi .
$$

As with the Dirac equation we choose units so that $\hbar=e=1$ where $e$ is the electric charge of the particle. We can write down an abstract version of this equation as follows:

$$
\left(i \frac{d}{d t}+V\right)^{2} \psi=\left(c^{2} A^{2}+m^{2} c^{4}\right) \psi
$$

where $A$ and $V$ are self-adjoint operators on some Hilbert space $\mathbb{H}_{0}$. The "free" equation is the case $V=0$ :

$$
d^{2} \psi / d t^{2}=-L(c)^{2} \psi
$$

where $L(c)=\left(c^{2} A^{2}+m^{2} c^{4}\right)^{1 / 2}$. The free equation can be solved explicitly and its behavior as $c \rightarrow \infty$ treated by means of the spectral theorem; we omit the easy details.

We turn now to a careful treatment of the abstract equation (4.2). Let $A$ and $V$ be self-adjoint operators on $\mathbb{H}_{0}$, and define $L(c)$ as above. Then (4.2) becomes

$$
\ddot{\psi}=-L(c)^{2} \psi+V^{2} \psi+2 i V \dot{\psi}
$$

We convert (6.7) into a first order system in the usual way by defining $\psi_{1}=\psi$, $\psi_{2}=\dot{\psi}$. Then we get

$$
\frac{d}{d t}\left[\begin{array}{l}
\psi_{1} \\
\psi_{2}
\end{array}\right]=\left(\left[\begin{array}{cc}
0 & I \\
V^{2}-L^{2} & 0
\end{array}\right]+\left[\begin{array}{cr}
0 & 0 \\
0 & -2 i V
\end{array}\right]\right)\left[\begin{array}{l}
\psi_{1} \\
\psi_{2}
\end{array}\right] .
$$

We want to interpret this rigorously on an appropriate Hilbert space, namely that given by the "energy" norm associated with the abstract Klein-Gordon equation (4.4).

As with the Dirac equation, we assume that $V$ is bounded relative to $A$. Then, when $c$ is sufficiently large, we will have the inequality

$$
\|V \psi\| \leqq \alpha\|L(c) \psi\|
$$

for all $\psi$ in $\mathscr{D}(A)=\mathscr{D}(L(c))$, where $\alpha$ can be made as small as we like. (Note: in the calculations that follow we will write $L$ for $L(c)$.) 
Although $V$ is a small perturbation of $L, V^{2}$ need not be bounded relative to $L^{2}$. However, $L^{2}-V^{2}$ can be interpreted as a self-adjoint operator using quadratic form perturbation theory (see [5] or [7]; a similar approach has been taken by Weder [13]). Indeed, we have

and, for $\psi$ in $2\left(L^{2}\right)$,

$$
\mathscr{Q}\left(L^{2}\right)=\mathscr{D}(L)=\mathscr{D}(A) \subseteq \mathscr{D}(V)=\mathscr{2}\left(V^{2}\right)
$$

$$
\left(V^{2} \psi, \psi\right)=(V \psi, V \psi) \leqq \alpha^{2}(L \psi, L \psi)=\alpha^{2}\left(L^{2} \psi, \psi\right)
$$

where we can assume that $\alpha<1$ by taking $c$ sufficiently large. Thus

$$
\left(1-\alpha^{2}\right) m^{2} c^{4}(\psi, \psi) \leqq\left(1-\alpha^{2}\right)\left(L^{2} \psi, \psi\right) \leqq\left(\left(L^{2}-V^{2}\right) \psi, \psi\right) \leqq\left(L^{2} \psi, \psi\right)
$$

in the sense of quadratic forms. Accordingly $\mathscr{D}(A)=\mathscr{Q}\left(L^{2}\right)$ is complete with respect to the inner product defined by $L^{2}-V^{2}$, and so there is a well-defined positive self-adjoint operator on $\mathbb{H}_{0}$, which we will denote by $M(c)^{2}$ or $\left(L(c)^{2}-V^{2}\right)$, such that $\mathscr{D}(M(c))=\mathscr{2}\left(L^{2}-V^{2}\right)=\mathscr{2}\left(L^{2}\right)=\mathscr{D}(L)$ and such that $M(c)^{2}=L(c)^{2}-V^{2}$ in the sense of quadratic forms.

Now form the Hilbert space $\mathbb{H}=\mathscr{D}(A) \oplus \mathbb{M}_{0}$ with the inner product

$$
\begin{aligned}
{[\psi, \psi]_{c} } & =\left(M(c)^{2} \psi_{1}, \psi_{1}\right)+\left(\psi_{2}, \psi_{2}\right) \\
& =\left(\left(L(c)^{2}-V^{2}\right) \psi_{1}, \psi_{1}\right)+\left(\psi_{2}, \psi_{2}\right) .
\end{aligned}
$$

With respect to this "energy" inner product, the operator

$$
S=S(c)=\left[\begin{array}{cc}
0 & I \\
-M(c)^{2} & 0
\end{array}\right]
$$

with domain $\mathscr{D}(S)=\mathscr{D}\left(M(c)^{2}\right) \oplus \mathscr{D}(A)$, is skew-adjoint. (This is a standard fact; cf. for example [2, Proposition 3.1].) Moreover the operator

$$
T=\left[\begin{array}{cc}
0 & 0 \\
0 & 2 i V
\end{array}\right],
$$

with domain $\mathscr{D}(T)=\mathscr{D}(A) \oplus \mathscr{D}(V)$, is also skew-adjoint.

Lemma 4.1. $T$ is bounded relative to $S(c)$, and the relative bound is less than 1 if $c$ is sufficiently large. Hence $S(c)+T$ is skew-adjoint if $c$ is sufficiently large.

Proof. If $\psi=\left(\psi_{1}, \psi_{2}\right)$ is in $\mathscr{D}(S)$, then $\psi_{2}$ belongs to $\mathscr{D}(A) \subseteq \mathscr{D}(V)$, so $\psi$ is in $\mathscr{D}(T)$. Moreover, by equation (4.6),

$$
\|T \psi\|^{2}=4\left\|V \psi_{2}\right\|^{2}=4\left(V^{2} \psi_{2}, \psi_{2}\right) \leqq 4 \alpha^{2}\left(L^{2} \psi_{2}, \psi_{2}\right)
$$

so that

$$
\|S \psi\|^{2} \geqq\left(M^{2} \psi_{2}, \psi_{2}\right)=\left(\left(L^{2}-V^{2}\right) \psi_{2}, \psi_{2}\right) \geqq\left(1-\alpha^{2}\right)\left(L^{2} \psi_{2}, \psi_{2}\right) .
$$

Thus $\|T \psi\|^{2} \leqq 4 \alpha^{2} /\left(1-\alpha^{2}\right)\|S \psi\|^{2}$. The constant here will be less than 1 when $c$ is large.

It follows that $S(c)+T$ generates a one-parameter group of operators on H which are unitary with respect to the inner product $[,]_{c}$. This inner product depends on $c$, and so it will be useful when examining the nonrelativistic limit 
to transform to a new Hilbert space with an inner product independent of $c$. (Veselić $[11,12]$ has a different approach, working on the Hilbert space $\mathscr{D}(A) \oplus \mathbb{H}_{0}$ with a fixed indefinite inner product related to the Klein-Gordon conserved density. His methods appear to require extremely strong conditions on the scalar potential $V$, and he does not consider magnetic vector potentials.)

Define $\mathbb{H}_{1}=\mathbb{H}_{0} \oplus \mathbb{H}_{0}$ with the direct sum inner product and define an operator $U(c)$ from $\mathbb{H}$ to $\mathbb{H}_{1}$ by

$$
U(c)=\frac{1}{\sqrt{2}}\left[\begin{array}{ll}
M(c) & -i I \\
M(c) & +i I
\end{array}\right]
$$

Note that we have

$$
U(c)^{-1}=\frac{1}{\sqrt{2}}\left[\begin{array}{cc}
M(c)^{-1} & M(c)^{-1} \\
i I & -i I
\end{array}\right] .
$$

One easily checks that $U(c)$ is unitary with respect to the inner product $[,]_{c}$ on $\mathbb{H}$ and the usual inner product on $\mathbb{H}_{1}$.

Now consider the self-adjoint operator $H(c)$ on $\mathbb{H}_{1}$ defined by

$$
i H(c)=U(c)(S(c)+T) U(c)^{-1} .
$$

We have $\mathscr{D}(H(c))=U(c) \mathscr{D}(S(c))=\mathscr{D}(A) \oplus \mathscr{D}(A)$, independent of $c$. Moreover we compute

$$
U(c) S(c) U(c)^{-1}=i\left[\begin{array}{cc}
M(c) & 0 \\
0 & -M(c)
\end{array}\right]=i H_{0}(c)
$$

and

$$
U(c) T U(c)^{-1}=i\left[\begin{array}{rr}
V & -V \\
-V & V
\end{array}\right]=i W .
$$

Thus we have the self-adjoint operator

$$
H(c)=H_{0}(c)+W=\left[\begin{array}{cc}
M(c)+V & -V \\
-V & -M(c)+V
\end{array}\right] .
$$

We can deduce the limiting behavior of $e^{i t H(c)}$ by methods similar to those employed in Sect. 2 for the Dirac equation.

Lemma 4.1. Given $f \in \mathscr{D}\left(A^{2}\right)$, there are vectors $f(c), g(c)$ such that, as $c \rightarrow \infty$, we have $f(c) \rightarrow f, g(c) \rightarrow 0$, and

$$
\left(H(c)-m c^{2}\right)\left[\begin{array}{l}
f(c) \\
g(c)
\end{array}\right] \rightarrow\left[\begin{array}{c}
H(\infty) f \\
0
\end{array}\right]
$$

where $H(\infty)=A^{2} / 2 m+V$.

Proof. We can take $f(c)=f, g(c)=-\left[M(c)+m c^{2}\right]^{-1} V f$. From (4.14), the first component of the left side of (4.15) is

$$
\left(M(c)-m c^{2}\right) f+V f+V\left[M(c)+m c^{2}\right]^{-1} V f,
$$


while the second component is $-V\left[M(c)+m c^{2}\right]^{-1} V f$. Thus we need to prove

and

$$
\left(M(c)-m c^{2}\right) f \rightarrow A^{2} f / 2 m
$$

$$
V\left[M(c)+m c^{2}\right]^{-1} V f \rightarrow 0 .
$$

Because $V$ is bounded relative to $A$ we have the inequality (in the sense of quadratic forms) for some constants $\alpha, \beta$ :

$$
V^{2} \leqq \alpha^{2} A^{2}+\beta^{2} .
$$

Accordingly,

$$
L(c)^{2}-\alpha^{2} A^{2}-\beta^{2} \leqq L(c)^{2}-V^{2}=M(c)^{2} \leqq L(c)^{2} .
$$

Hence, for $c$ large (so that $m^{2} c^{4}>\beta^{2}$ and $c^{2}>\alpha^{2}$ ), we have

$$
\left(c^{2}-\alpha^{2}\right) A^{2} \leqq M(c)^{2} .
$$

From this it follows that $\left\|A M(c)^{-1}\right\| \leqq\left(c^{2}-\alpha^{2}\right)^{-1 / 2}$ and a fortiori $\| A[M(c)+$ $\left.m c^{2}\right]^{-1} \| \leqq\left(c^{2}-\alpha^{2}\right)^{-1 / 2}$.

Hence, for $f \in \mathscr{D}(A)$, we have

$$
\begin{aligned}
\left\|V\left[M(c)+m c^{2}\right]^{-1} V f\right\| & \leqq \alpha\left\|A\left[M(c)+m c^{2}\right]^{-1} V f\right\|+\beta\left\|\left[M(c)+m c^{2}\right]^{-1} V f\right\| \\
& \leqq\left[\alpha\left(c^{2}-\alpha^{2}\right)^{-1 / 2}+\beta\left(m c^{2}\right)^{-1}\right]\|V f\| .
\end{aligned}
$$

This establishes (4.17).

Turning to (4.16), we begin with the formula $M(c)^{2}-m^{2} c^{4}=\left(c^{2} A^{2}-V^{2}\right)$ (form sum) so that

$$
M(c)-m c^{2}=\left[M(c)+m c^{2}\right]^{-1} c^{2} A^{2}-\left[M(c)+m c^{2}\right]^{-1} V \cdot V
$$

where the right side is now an operator sum. Moreover $\left[M(c)+m c^{2}\right]^{-1} V$ is a bounded operator with norm equal to that of its adjoint, namely, as calculated above,

$$
\left\|V\left[M(c)+m c^{2}\right]^{-1}\right\| \leqq \alpha\left(c^{2}-\alpha^{2}\right)^{-1 / 2}+\beta\left(m c^{2}\right)^{-1} .
$$

This tends to 0 as $c \rightarrow \infty$. Hence in (4.19) we are left with the term $\left[M(c)+m c^{2}\right]^{-1} c^{2} A^{2}$.

We claim that $c^{2}\left[M(c)+m c^{2}\right]^{-1} \rightarrow 1 / 2 m$ strongly as $c \rightarrow \infty$. Indeed, from the inequalities (4.18) together with the formula for $L(c)^{2}$ we get

$$
m^{2} c^{4}-\beta^{2} \leqq M(c)^{2} \leqq m^{2} c^{4}+c^{2} A^{2}
$$

so that we have

$\left[\sqrt{m^{2} c^{4}+c^{2} A^{2}}+m c^{2}\right]^{-1} \leqq\left[M(c)+m c^{2}\right]^{-1} \leqq\left[m c^{2}+{\sqrt{\left.m^{2} c^{4}-\beta^{2}\right]}}^{-1}\right.$.

Here we have made use of the monotonicity of the square root on positive operators [7, Theorem V. 4.12].

From inequalities (4.20) it follows easily that $c^{2}\left[M(c)+m c^{2}\right]^{-1} \rightarrow 1 / 2 m$ weakly as $c \rightarrow \infty$. But since the lim sup of the norm of $c^{2}\left[m c^{2}+M(c)\right]^{-1}$ is $1 / 2 m$, weak convergence implies strong convergence. 
We may finish the discussion of the abstract Klein-Gordon equation along the lines of our treatment of the abstract Dirac equation. Thus we may introduce the spectral projections $P_{+}(c), P_{-}(c)$ associated with $H(c)$ and prove that $P_{ \pm}(c) \rightarrow P_{ \pm}$strongly as $c \rightarrow \infty$ by the argument used in Sect. 4 .

Moreover "magnetic potentials" can be introduced by altering the operator $A$, just as in the Dirac case. The Klein-Gordon operator is slightly more complicated; however, if for example $A^{2}=A_{1}^{2}+A_{2}^{2}+A_{3}^{2}$ then we introduce magnetic potentials $B_{1}, B_{2}, B_{3}$ so that our new $A$ is given by

$$
\left(A^{\prime}\right)^{2}=\left(A_{1}-B_{1}\right)^{2}+\left(A_{2}-B_{2}\right)^{2}+\left(A_{3}-B_{3}\right)^{2}
$$

in the sense of quadratic forms. We want the perturbation $\mathbf{B}=\left(B_{1}, B_{2}, B_{3}\right)$ to be such that $\mathscr{D}\left(A^{\prime}\right)=\mathscr{D}(A)$. A simple estimate using quadratic forms shows that this is guaranteed provided $B=\left(\sum_{i} B_{i}^{2}\right)^{1 / 2}$ is bounded relative to $A$ with relative bound less that 1 ; this is essentially the same condition as in the Dirac case.

The following theorem summarizes the main results.

Theorem 4.2. Let $A$ and $V$ be self-adjoint operators on a Hilbert space $\mathbb{H}_{0}$ where $V$ is bounded relative to $A$. Define $M(c)$ by the quadratic form relation $M(c)^{2}=$ $m^{2} c^{4}+c^{2} A^{2}-V^{2}$. Let $H(c)$ be defined on $\mathbb{H}_{1}=\mathbb{H}_{0} \oplus \mathbb{H}_{0}$ by

$$
H(c)=\left[\begin{array}{cc}
M(c) & 0 \\
0 & -M(c)
\end{array}\right]+\left[\begin{array}{rr}
-V & V \\
V & -V
\end{array}\right] .
$$

Then, for c sufficiently large, $H(c)$ is self-adjoint and its spectrum has a gap containing 0 . The corresponding spectral projections $P^{ \pm}(c)$ converge strongly to $P^{ \pm}=\left[\begin{array}{ll}1 & 0 \\ 0 & 0\end{array}\right]$, $\left[\begin{array}{ll}0 & 0 \\ 0 & 1\end{array}\right]$ as $c \rightarrow \infty$. Moreover, if $H_{\infty}=A^{2} / 2 m+V$, we have

$$
e^{\mp i t m c^{2}} e^{i t H(c)} P^{ \pm}(c) \rightarrow e^{ \pm i t H^{\infty}} P^{ \pm}
$$

strongly, uniformly on bounded t-intervals, as $c \rightarrow \infty$.

Acknowledgments. We thank the referee for some useful suggestions which shortened our arguments.

\section{References}

1. Chernoff, P. R. : Essential self-adjointness of powers of generators of hyperbolic equations. J. Funct. Anal. 12, 401-414 (1973)

2. Chernoff, P. R. : Schrödinger and Dirac operators with singular potentials and hyperbolic equations. Pac. J. Math. 72, 361-382 (1977)

3. Cirincione, R. J.: A mathematical study of the non-relativistic limit in quantum mechanics. Ph.D. Thesis University of California, Berkeley (1979)

4. Davies, E. B. : Asymptotic analysis of some abstract evolution equations. J. Funct. Anal. 25, $81-101$ (1977)

5. Faris, W. G. : Self-adjoint operators. In : Lecture notes in mathematıcs, vol. 433. Berlin, Heidelberg, New York: Springer 1975

6. Hunziker, W. : On the nonrelativistic limit of the Dirac theory. Commun. Math. Phys. 40, 215-222 (1975) 
7. Kato, T.: Perturbation theory for linear operators, (2nd ed.) Berlin, Heidelberg, New York: Springer 1975

8. Palais, R. S. : Differential operators on vector bundles. In: Seminar on the Atiyah-Singer index theorem. Annals of Mathematics Studies, No. 57. Princeton: Princeton University Press 1965

9. Prosser, R. : Relativistic potential scattering. J. Math. Phys. 4, 1048-1054 (1964)

10. Reed, M., Simon, B. : Methods of modern mathematical physics, Vol. 2. New York: Academic Press 1975

11. Veselić, K.: A spectral theory for the Klein-Gordon equation with an external electrostatic potential. Nucl. Phys. A 147, 215-224 (1970)

12. Veselić, K. : Perturbation of pseudoresolvents and analyticity in $1 / \mathrm{c}$ in relativistic quantum mechanics. Commun. Math. Phys. 22, 27-43 (1971)

13. Weder, R. : Selfadjointness and invariance of the essential spectrum of the Klein-Gordon equation. Helv. Phys. Acta 50, 105-115 (1977)

14. Wolf, J. A. : Essential self-adjointness of the Dirac operator and its square. Indiana Univ. Math. J. 22, 611-640 (1973)

Communicated by B. Simon

Received January 21, 1980; in revised form June 18, 1980 